\title{
Philosophiques
}

\section{Jean-Luc Marion, Étant donné. Essai dune phénoménologie de la donation, Paris, PUF, Épiméthée, 1997, 452 p.}

\section{Martin Gagnon}

Volume 25, numéro 1, printemps 1998

URI : https://id.erudit.org/iderudit/027481ar

DOI : https://doi.org/10.7202/027481ar

Aller au sommaire du numéro

Éditeur(s)

Société de philosophie du Québec

ISSN

0316-2923 (imprimé)

1492-1391 (numérique)

Découvrir la revue

Citer ce compte rendu

Gagnon, M. (1998). Compte rendu de [Jean-Luc Marion, Étant donné. Essai dune

phénoménologie de la donation, Paris, PUF, Épiméthée, 1997, 452 p.]

Philosophiques, 25(1), 136-139. https://doi.org/10.7202/027481ar d'utilisation que vous pouvez consulter en ligne.

https://apropos.erudit.org/fr/usagers/politique-dutilisation/ 
Jean-Luc Marion, Étant donné Essai d'une phénoménologie de la donation, Paris, PUF, Épiméthée, 1997, 452 p.

Bien qu'on ne puisse rien présumer cle l'itinéraire futur de la pensée de Jean-Luc Marion, force est de reconnaître que Étant donné s'impose d'emblée comme un elefd'œuvre: la force, l'originalité et la rigueur qui se flégagent de la plupart des analyses phénoménologiques cléveloppées dans cet ouvrage - pour ne rien dire de la justesse des incursions historiques qui viennent appuyer ou illustrer ces analyses - nous invitent cn effet à ranger Étant donné parmi les "faits d'armes "les plus audacieux et les plus féconds de la philosophie française cles vingt dernières années.

En 1989, la publication de Réduction et donation, "ouvrage strictement de méthode " $(E D, 17)$, avait déjà suscité dans la communauté philosophique française de vifs débats autour des possibilités et des limites de la phénoménologie. Marion se 
proposait alors de montrer que la richesse de ce qui est donné varie toujours en fonction de la profondeur de la réduction effectuée : la phénoménologie ne réalise sa possibilité ultime qu'à la condition de reconduire, en deçà cles horizons arbitrairement imposés de l'objectité (Husserl) et de l'étantité (Heidegger), à la forme pure de l'appel seule réduction ton "réductrice" parce que coincidant avec un maximum de phénoménalité, voire avec la phénoménalité elle-même, tant et pour autant qu'il n'est plus aucune décision ni aucun horizon pour compromettre la manifestation cle celle-ci en l'assujetissant de l'extérieur à des exigences qui lui sont étrangères.

Étant donné vient enrichir sans la détourner la problématique ouverte par Réduction er donation. Nous nous contenterons ici tle dégager les principales lignes de force de cel corrichissement à partir dles quatre grandes thèses qui, de manière plus ou moins explicite, nous apparaissent commander le développement de cet ouvrage.

1) Ce qui se montre doit d'abord se donner. Telle est la thèse qui, de l'aveu de l'auteur, constifue l'a unique thème » $\{\mathrm{RD}, 10\}$ de l'ouvrage. Qu'est-ce done qui donne au phénomène d'être phénomène el ainsi de se montrer? De quoi le phénomène tiret-il sa manifestation? De ce qu'il se domne, d'abord et avant tout. Qu'il soit toujours possible de concevoir certains phénomènes comme s'exceptant de l'horizon de l'objeclité, voire de l'étantifé, il n'est cependant aucun phénomène (pas même celui du néant) qui ne puisse s'excepter de l'horizon - si tant est qu'on puisse encore parler ici d'horizon - de la donation, e'est-à-clire de ce qui permet à tout phénomène d'apparaître littéralement comme un pur donné, voire un pur don. La visée ultime de toute réduction pliénoménologique rigoureusement effectuée consisterait par conséquent à reconduire à ce don que tout phénomène constitue du fait de sa dépendance originaire (el tout à fait irrécluctible) à l'égard du processus de la donation.

$\hat{\Lambda}$ qui soupçonnerait que la thèse de l'universalité de la donation puisse ici profiter de l'ambivalence sémantique du terme même de " clonation ", Marion répond que cette ambivalence esl " un fait [dont l'admission] n'implique nulle exploitation" IRD, 91). Que l'arlnission non eritique de ce "fait "langagier soit légitime ou non, il n'eu rlemeure pas moins ru'elle rend possible une toute nouvelle interprétation du privilege traditionnellement reconnu à l'intuition au sein de la pensée phénoménologigue. En effet, ce privilège ne vaut qu'en autant que l'intuition demeure donatrice : " (ue nous imporlerait une intuition el quelle autorité lui reconmaitrionsnous, si elle ne nous donnai rien $[. .$. ? " (RD, 27). Mais que re (jui se montre doive d'abord se donner ne semble pas exiger', à l'inverse, que ee qui se clonne doive nécessairement se montrer : "selon notre thèse de fond, la donation n'équivaut pas à l'intuition et ne l'exige pas nécessarement" (RD, 82 ; nous soulignons). On notera cependant une certaine Iension clans le rliscours cle Marion entourant la nécessité ou la non-nécessilé, pour ce qui se donne, de se montrer. Cette tension apparaît de façon particulièrement frappante lorsque l'auteur s'avise le ce que le concept de donation implicpue bel el bien (serait-ce d'ailleurs là un don inaperçu de son ambivalence sémantique ?) un " donner à " : cle fait o comment la donation pourrait-elle donner un donné, sans le lui |sic| faire voir, donc sans l'adresser à quelque instance comme une "conscience" "? "(RD, 101). Et Marion de conclure, négligeant la nuance introduite plus haut, que non sculement le phénonène peut se montrer, mais qu'il le doit (RD, 102).

2) Ce qui se donne ne dépend que de soi-même pour se dormer. Si la réduction ne réduit bien qu'à la donation, seul ce qui se donne à partir de soi peut décider de la phénoménalité et de ses modes possibles. Le centre de gravité phénoménologique se cléplace : le phénomène n'est plus fonction d'un " je pense ", d'un ego, voire d'un Dasein qui décréteraient à l'avance les conditions que ce phénomène doit satisfaire pour obtenir droit de cité en philosophie. Désormais, le phénomène n'est plus fonction que de sa seule automanifestation : plus rien ne décide de son apparition que 
le " soi " qui lui est propre. Tel est, selon Marion, le dernier principe, que " rien ne précède le phénomène, sinon sa propre apparition à partir de soi " $(\mathrm{RD}, 29)$.

Ce réplacement ne va pas sans entraîner certaines conséquences pour ce qui regarde le statut du sujet phénoménologique : la donation de soi du phénomène implique rigoureusement que la subjectivité se clélinisse comme donation à soi, c'est-àdire conme recevant sa délermination et sa situation du déploiement du phénomène lui-même. Ce n'est donc plus le phénomène qui se soumet aux conditions de la subjectivité, mais au contraire la subjectivité qui se plie à la logieque apophantique du phénomène. Le "sujet" est moins substrat qu'organe de monstration de ce qui se donne: il n'est ni plus ni moins qu'un « attributaire a dont la situation ne se concrétise qu'en rapport à la charge incirlentelle et événenentielle qui cararactérise tout phénomène de droit commun. S'inspirant ici d'un procédlé pictural, Marion décril sous le titre d'anamorphose cette nise en situation de la subjectivité à partir du mode de manifestation du phénomène. Ainsi, pour accédler à la forme d'arrivée du phénomène, la subjectivité doit "renoncer à organiser la visibilité à partir du libre choix ou du site: propre d'un spectateur désengagé, pour se la laisser dicter par le phénomène luiniême, en $[$ son $]$ soi " $(\mathrm{RD}, 176)$.

3) Ce qui se dome donne porfors phus quil nest possible de woir. Tel est le cas lorsque la subjectivité ne parvient plus à maintenir dans les limites de ses visées intentionnelles la richesse intuitive de ce qui lui est domé. L'aceueil que réserve Marion à l'examen de cette possibilité manifeste on ne peut plus clairement la distance prise à l'égard du "principe des principes ou l'intuition deneure sournise une logirgue de la pénurie " (RD, 259) exigeant que l'intuition se produise à l'intérieur de l'horizon du comnu ot qu'elle ne transgresse jamais les ressources constituantes rlu Je transcendantal. Le " dernier principe "prend donc ici la relève du "prineipe des principes "afin de légitimer phénoménologiquement la possibilité d'une donation à ce point salurée d'intuition qu'elle "surpasse l'intention " (RD, 315) el défie toute anticipation subjective cle même que toute pré-vision théorétique.

Reprenant certaines analyses exposées ailleur's ivoir "Le phénomène saturé " rlans Phénoménologie et théologie. Paris, Critcrion, Itcées, 1992, [extraits : p. 79-128]), Marion entreprend de dégager les diverses modalités de saturation plénoménale en s'appuyant sur le tableau kantien des ratégories. Ainsi, a invisable selon la qualité, insupportable selon la qualité, absolu selon la relation, irregardable selon la morlalité " $(\mathrm{RD}, 280$. le phénonène saluré montre que l'intuilion peut aussi obéir à une logigue de l'excédent. Que ce soit sous la guise de l'événement historique, de l'idole, du rableau, de la chair ou de l'icône, le phénomène saturé illustre par excellence les possibilités ultimes de la phénoménologic et soulève par conséçuent la question de savoir " jusqu’à quel degré la saturation peut se déployer " (RD, 326) lout en restant phénomène. La réponse coüncide avec le phénomène de révélation, lecfucl ouvre la possibilité d'une saturation extrême, sursaturée dlans la mesure où les quatre possibilités de saturation s'y précipitent et se déterminent réciproquement pour " [saturer] la phénoménalité au second degré, par saturation de saturation " ( $R D, 327$ ).

4) Ce qui se donne ne se montre le plus souvem qu' à qui accepte de recevoir avant de voir. La problématique du phénomène saturé libère la possibilité d'un écart linite entre la donation et la monstration. Dès lors que ce qui est donné excède en intuition la viséc et le concept, l'impact de la donation se déploie sous la forme d'un appel : la monstration de ce qui est donné repose alors sur une décision de la part de la "subjectivité " de prendre sur' soi cet appel, de s'en faire le témoin répondant, l'adonné : a nous ne voyons sans les vouloir que les phénomènes les plus pauvres [...] dès qu'un phénomène s'enrichit en intuition, done dès que son degré de clonation s'accroît, il nous faut $[. .$.$] le constituer et le supporter pour le voir... " (RD, 420). La$ possibilité du voir repose donc ici sur' l'effectivité d'une décision de recevoir qui peut 
ne pas se produire. L'excès du don peut par conséquent se donner sous le mode de l'abandonné : plutôt que d'accepter le don, l'adonné décide de se dérober à sa surcharge intuitive.

Si Marion montre, de façon assez convaincante, qu'il est de ces " phénomènes" dont la manifestation s'appuie à une décision préalable de la part de l'adonné, on peut cepenclant douter du succès de ses efforts en vue de préserver la pureté phénoménologique de ces descriptions de toute "contamination " au contact de l'éthique. Ainsi, lorsque l'auteur entreprend d'approfondir la problématique de l'abandon à parlir du regard d'autrui el qu'il qualifie de "mépris " le fait de refuser de soutenir ce regard, de le "prendre en vue ", sa volonté de soustraire le concept de mépris à toute interprétation élhique le conduit, contre toute attente, à faire du mépris la condition même de la sociabilité dans la mesure où il nous clispense a de voir l'autre lomme. pour ce qu'il se donne " (RD, 137). Certes que l'anonymat et l'interchangeabilité des "persomes" soient le fait de la quolidienneté banale, que ce titre "l'autre homme" apparaisse d'aborl à travers sa fonction d'agent social, économicue, etc. n'implique pas nécessairement que celui-ci lasse l'objet de mépris, c'est-à-dire qu'il disparaisse sous celte fonction au point de s'y réduire. En diluant comme il le fait le concept de mépris, Marion finit bien par le libérer (le toute connotation éthique, mais c'est qu'il n'entend rien de plus par "mépris " que cette sociabilité commune et inauthentique que Heidegger a déjà ressaisie sous le mode du On.

Je ne ne saurais conclure celte présentation sans signaler le point sans doute le plus névralgique de cet ouvrage. Toutes les démonstrations de Marion découlent d'une prémisse fondamentale : le phénomène se clonne. De l'aveu de l'auteur lui-même, il n'est rien de plus embarrassant que le " soi " lu phénomène : Reste à franchir le pas le plus périlleux : penser ce se/soi - qui seul permet au phénomène de se montrer" (RD, 439-440). Il resterait surtout à se demander si ce pas peut être franchi de l'intérieur de la phénoménologie. Certes, qu'il faille penser ce soi, la tradition phénonénologique française nous y invite déjà clepuis belle lurette : Merleau-Ponty, Lévinas, Ifenry - et Marion à leur suite - nous ont depuis Iongtemps familiarisés avec ces inversions et ces bouleversements re l'intentionnalité qu'entrainent les expériences de "phénonèmes saturés " et foute expérience analogue où la subjectivité se découvre divantage visée (que visante, clavantage interrogée qu'interrogeante. Tout se passe alors effectivement comme si un "Autre " prenait de lui-mêne initiative de se clonner, de se montrer. Nul doute que la plénoménologie soit parfaitement légitimée à clécrire ce " comme si ". l.a question est seulement de savoir si les phénoménologues 'ux-mêmes peuvent s'accommorler de cette "limite "ê éviter, conformément à leurs précautions méthodologigues les plus récurrentes, de répondrè à un appel autrement plus puissant que celui de l'Être, de la Vie ou de l'Autre - soit l'appel de la métaphysique elle-même.

\section{Martin Gagnon}

Département de philosophie

Université de Montréal 